\title{
Thermodynamics and non-Gaussian Measures in the Covariant Variational Approach to Yang-Mills Theory
}

\section{Markus Quandt*}

E-mail: markus .quandteuni-tuebingen. de

\section{Hugo Reinhardt}

E-mail: hugo.reinhardt@uni-tuebingen.de

\section{Davide Campagnari}

E-mail: davide. campagnarieuni-tuebingen. de

Institute for Theoretical Physics

University of Tübingen

Auf der Morgenstelle 14

72076 Tübingen, Germany

\begin{abstract}
The covariant variational approach to Yang-Mills theory is further developed. After discussing the foundations of the method both at zero and finite temperature, we briefly recall the effective action for the Polyakov loop and the critical properties of the deconfinement phase transition within this approach. The thermodynamics of pure Yang-Mills theory are studied in detail and the resulting equation of state is compared to lattice data. While there is good agreement in the deconfined (high-temperature) phase, a small but non-zero pressure is predicted in the confined phase at low temperatures, in contrast to physical expectations. We propose possible improvements to address this issue. Finally, we discuss the combination of the variational approach with Dyson-Schwinger techniques and argue that the method can be used as a tool to determine the optimal vertices for a truncated set of Dyson-Schwinger equations. We briefly lay out how this technique could be applied to Yang-Mills theory at zero temperature.
\end{abstract}

XIII Quark Confinement and the Hadron Spectrum - Confinement 2018

31 July - 6 August 2018

Maynooth University, Ireland

\footnotetext{
* Speaker.
} 


\section{Introduction}

The low energy sector of quantum chromodynamics (QCD) and, in particular, its phase diagram are among the most actively researched topics in elementary particle physics. Experimentally, heavy ion collisions at the large hadron collider (LHC) are beginning to probe strongly interacting matter at such high temperatures and baryon densities that the properties of the resulting quarkgluon plasma can be studied in detail. By contrast, theoretical investigations of the full QCD phase diagram through lattice simulations are still restricted to rather small densities due to the sign problem. Alternative functional methods in the continuum are therefore very welcome. In covariant gauges, the most widely used methods are functional renormalization group (FRG) flow equations [1] and Dyson-Schwinger equations (DSE) [2], while extensions of the Faddeev-Popov action through mass terms [3] or the Gribov-Zwanziger term [4] are also proposed. If we dispense with manifest covariance, the Hamiltonian approach to QCD in Coulomb gauge [5] provides a concise and physically transparent approach to QCD using variational techniques.

In a series of papers $[6,7,8,9]$, we have proposed an alternative continuum method which combines the benefits of a variational approach with the simplicity of a manifestly covariant setup. Starting from Ansätze for the Euclidean path integral measure, the method leads to a set of integral equations for the low-order Green's functions that can be conventionally renormalized. In this talk, I will briefly discuss the fundamental ideas behind the variational approach and show that it gives an excellent description of the Yang-Mills propagators at zero temperature, while the extension to finite temperature can easily be achieved by standard techniques. I will then present results for the effective action of the Polyakov loop, as well as the pressure and energy density of the gluon plasma at finite temperature. While most thermal properties are correctly predicted (even quantitatively), the pressure and energy density in the confined phase show significant deviations, for which I propose possible improvements. Finally, I will give an outlook of how the variational approach can be extended beyond the Gaussian Ansatz, and how this technique can be used to optimize vertices in truncated DSEs.

\section{Foundations of the covariant variational approach}

Consider a theory of a quantum field $A(x)$ which is described by a Euclidean action $S[A]$. We will call $S[A]$ the target action of the variational principle, to distinguish it from the trial action $R[A]$ introduced in the variational ansatz

$$
d \mu_{R}=N_{R} \cdot d A \exp (-R[A])
$$

where $N_{R}$ it a normalization constant that makes $d \mu_{R}$ a probability measure on function space. Intuitively, we think of $R[A]$ as a simplified version of the original action, with variational parameters $\{\gamma\}$ that help to mitigate the error due to the replacement $S \rightarrow R$. More precisely, the variational parameters $\{\gamma\}$ in the ansatz $R[A]$ are determined by the condition that the free action

$$
\begin{aligned}
F\left(\mu_{R}\right) & =\langle S[A]\rangle_{\mu_{R}}-\mathrm{W}\left(\mu_{R}\right) \\
\mathrm{W}\left(\mu_{R}\right) & =\langle R[A]\rangle_{\mu_{R}}-\ln N_{R}
\end{aligned}
$$




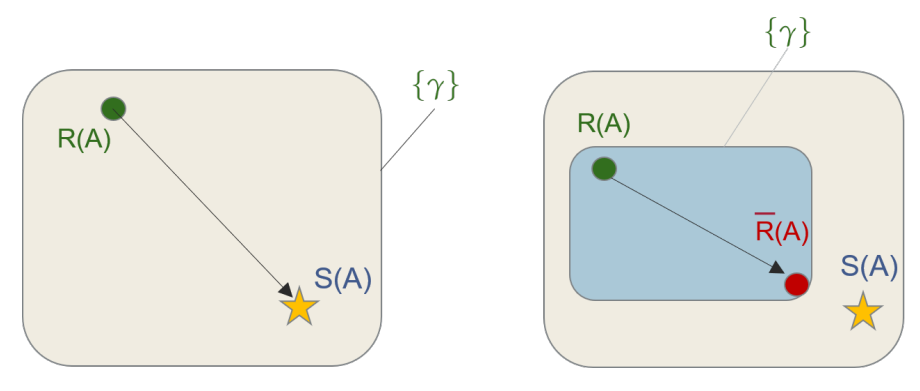

Figure 1: Schematic description of the variation principle in quantum field theory.

is minimized. This minimization is based on the similarity of the Euclidean field theory with statistical mechanics, and $\mathrm{W}(\mu)$ corresponds to the entropy of quantum fluctuations in the measure $\mu$. It is easy to see that the unique solution to the minimization problem (2.2) is simply the original Euclidean path integral measure, i.e. $R=S$. This is illustrated in the left panel of 1: the otpimal choice for the parameters $\{\gamma\}$ in the trial ansatz $R[A]$ is such that $R[A]=S[A]$. So it seems that the principle (2.2) is trivial, as its solution merely leads us back to where we started.

In reality, however, our limited computational power means the the set of measures $\{R[A]\}$ which we can handle is restricted and does not include the original target action $S[A]$ (or we could have solved the theory directly). This is depicted in the right panel of 1: now the parameters $\{\gamma\}$ in the trial ansatz $R[A]$ are non-trivial and lead to an optimal action $\bar{R}[A]$ within our computational reach, which is as close as possible to the target action. The more truncated our ansatz space, the less trivial will be the gap equations determining the variational parameters $\{\gamma\}$, since they have to make up for the severely restricted ansatz space.

Usually, the minimization of the free action is carried out in a restricted form, where the expactation value of the field is fixed to some prescribed value $\mathscr{A}$,

$$
F(\mu, \mathscr{A})=\left\{F(\mu) \mid\langle A\rangle_{\mu}=\mathscr{A}\right\} .
$$

Once this is minimized with respect to $\mu$, the minimizing measure $\mu_{\mathscr{A}}$ will implicitly depend on the prescribed field and the value of the minimal free action thus also becomes a functional of the external field, $\Gamma[\mathscr{A}]=F\left(\mu_{\mathscr{A}}, \mathscr{A}\right)$. Without restriction on the variational ansatz, this quantity is simply the quantum effective action, i.e. the generating functional of the proper functions of the original theory $S[A]$.

For Yang-Mills theory in the continuum, we must fix the gauge and the resulting FaddeevPopov (FP) action is not of the standard form (2.1). The FP determinant $J[A]$ can, however, easily be incorporated in the entropy $\mathrm{W}(\mu)$, so that the following modified free action is appropriate:

$$
F\left(\mu_{R}, \mathscr{A}\right)=\left\{\left\langle S_{\mathrm{gf}}\right\rangle_{\mu_{R}}-\left[\langle R\rangle_{\mu_{R}}+\langle\ln J\rangle_{\mu_{R}}-\ln N_{R}\right] \mid\langle A\rangle_{\mu_{R}}=\mathscr{A}\right\} .
$$

\section{Zero temperature: the Gaussian ansatz}

In the following, we employ Landau gauge $\partial_{\mu} A_{\mu}=0$ and restrict our trial measure to the simplest choice, namely the set of modified Gaussian measures with arbitrary kernel $\omega$,

$$
d \mu[A]=N(\omega) \cdot d A J[A]^{-1} \exp \left[-\frac{1}{2} \int d(x, y) A_{\mu}^{a}(x) \omega_{\mu v}(x, y) \delta^{a b} A_{v}^{b}(y)\right]
$$



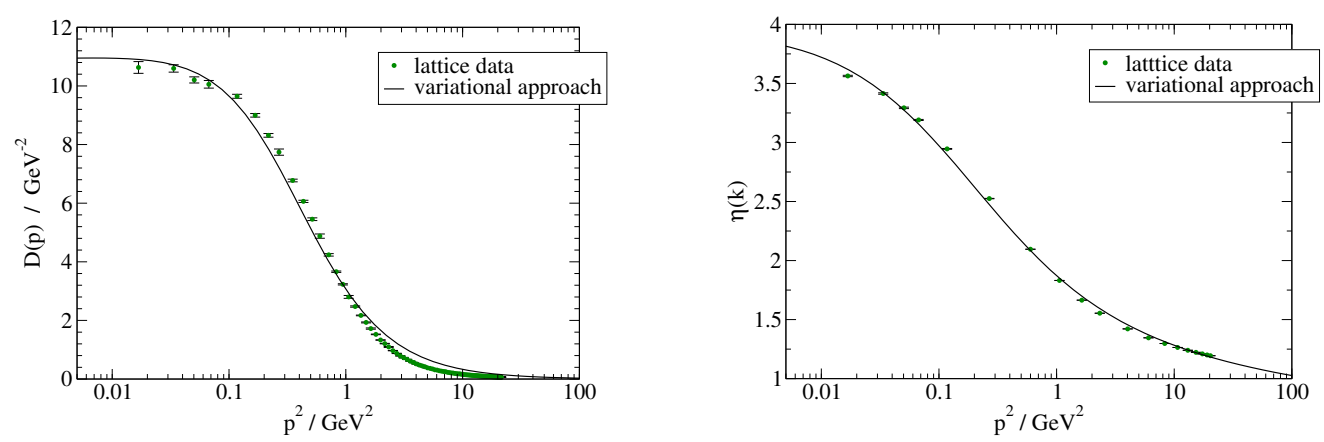

Figure 2: Gluon propagator (left) and ghost form factor (right) at $T=0$ from the decoupling solution of the variational approach, compared to high-precision lattice data taken from Ref. [10].

The physical concept behind this choice is the notion of a weakly coupled constituent gluon with an enhanced weight near the Gribov horizon (where $J(A)=0$ ). After Fourier transformation, the variational kernel $\omega_{\mu v}(k)=\left(\delta_{\mu v}-k_{\mu} k_{v} / k^{2}\right) \omega(k)$ becomes the inverse gluon propagator. Inserting eq. (3.1) into eq. (2.4) and minimizing w.r.t. $\omega(k)$ yields a closed system of integral equations which can be written in diagramatical form as

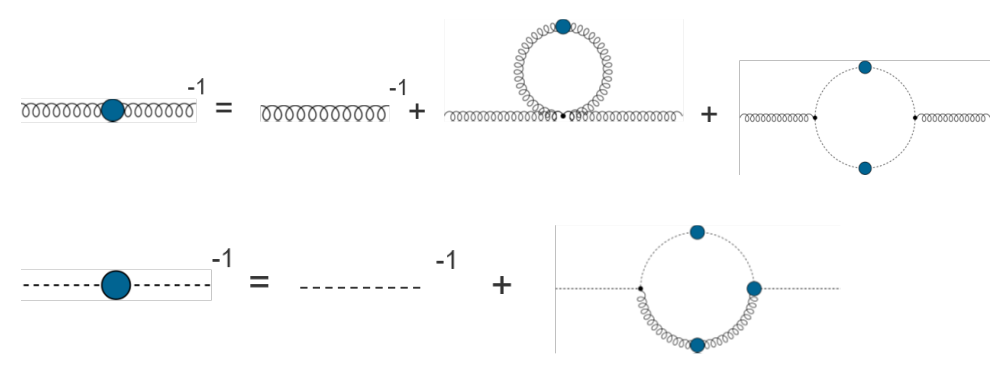

The ghost propgator in the second line emerges from the ghost representation of the FP determinant. In principle, we would also have to list the ghost-gluon vertex as a variational kernel. It is, however, well known to receive very little dressing even in the confined phase, and we simply assume the bare solution for its gap equation (rainbow-ladder approximation). The system (3.2) can be conventionally renormalized by adding three counter terms

$$
\mathscr{L}_{\mathrm{CT}}=\delta Z_{\mathrm{A}} \cdot \frac{1}{4}\left(\partial_{\mu} A_{v}^{a}-\partial_{v} A_{\mu}^{a}\right)^{2}+\delta M^{2} \cdot \frac{1}{2}\left(A_{\mu}^{a}\right)^{2}+\delta Z_{c} \cdot \partial_{\mu} \bar{c} \partial^{\mu} c .
$$

The coefficients are determined by three renormalization conditions which are imposed at three different scales for numerical stability. In particular, the mass counter term is fixed by a condition $\omega\left(\mu_{0}\right)=Z M_{A}^{2}$ which looks similar to a gluon mass term. However, since $\mu_{0}>0$ it merely represents a dimensionfull mass parameter which sets the overall scale. Indeed, the system (3.2) admits scaling solutions which describe a massless gluon while still having $M_{A} \neq 0$. As can be seen from Fig. 2, the decoupling solution with a finite ghost form factor at $\mu=0$ describes the lattice data much better and offers an almost perfect quantitative agreement. It should be noted that the renormalization determines all free parameters uniquely at $T=0$. All subsequent calculations at $T>0$ re-use these settings and therefore lack any adjustable parameters. 


\section{Finite temperature and the Polyakov Loop}

Since our trial measure induces a conventional Euclidean quantum field theory, finite temperature can be introduced as usual by compactifying the Euclidean time direction to a circle of length $\beta=T^{-1}$, and imposing periodic boundary conditions for the gluons and the ghosts (even though the latter are fermions). One immediate consequence of this prescription is that the loop integration over the temporal component $k_{0}$ decays to a sum over Matsubara frequencies. Moreover, the heat bath breaks Lorentz covariance, and the temporal direction is also singled out in the Lorentz structure of the gluon propagator, which now acquires two separate variational kernels $\omega_{\|}(k)$ and $\omega_{\perp}(k)$ parallel and perpendicular to the heat bath, respectively. Despite these changes, the modifications to the propagators at finite temperature are rather modest: with increasing temperature, there is a slight enhancement of the ghost form factor and a mild suppression of the gluon propagator, with a somewhat larger temperature sensitivity in the direction longitudinal to the heat bath [7]. This is all in qualitative agreement with lattice simulations. In particular, there is no abrupt change in the qualitative behaviour of the propagators, and no sign of a phase transition.

In order to study confinement, we must hence look at a different observable. The order parameter for confinement in Yang-Mills theory is the Polyakov-loop winding around the compactified Euclidean time direction:

$$
\mathrm{L}(\mathbf{x}) \equiv \mathrm{P} \exp \left[-\int_{0}^{\beta} d t A_{0}(t, \mathbf{x})\right] .
$$

It is related to the free energy $F_{q}$ of a static quark, $\langle\operatorname{trL}(\mathbf{x})\rangle=\exp \left[-\beta F_{q}(\mathbf{x})\right]$, so that $\langle\operatorname{trL}(\mathbf{x})\rangle=0$ implies $F_{q}=\infty$ and hence static quark confinement, while $\langle\operatorname{trL}(\mathbf{x})\rangle \neq 0$ means $F_{q}<\infty$ and hence deconfinement. Some gauges admit a slightly different order parameter $\langle\operatorname{tr} \ln \mathrm{L}\rangle$, which for $G=$ $S U(2)$ reduces to the normalized quantity

$$
x \equiv \frac{\beta\left\langle A_{0}^{3}\right\rangle}{2 \pi}=\frac{\beta \mathrm{a}}{2 \pi} \in[0,1],
$$

parametrizing the Weyl chamber, while for $G=S U(3)$, we have two such variables $x$ and $y$ for the two Cartan directions $T^{3}$ and $T^{8}$. Two gauges for which eq. (4.2) has been shown rigorously [12] to be an order parameter for confinement are the Polyakov gauge, $\partial_{0} A_{0}=A_{0}^{1,2}=0$, and the background gauge

$$
A_{\mu}=\text { a } \delta_{\mu 0}+Q_{\mu}, \quad\left[D_{\mu}(\mathrm{a}), Q_{\mu}\right]=0 .
$$

In order to derive the effective action for the Polyakov loop, we would now have to go to background gauge and modify our Gaussian ansatz such that $\left\langle A_{\mu}^{3}\right\rangle=\delta_{\mu 0}$ a. Fortunately, we do not need to go through the entire calculation again. Using special properties of the Gaussian ansatz, one can show [8] that the variational kernel $\omega(k)$ from the Landau gauge calculation can be transferred to background gauge by simple replacements and shifts. Thus applying the rules to the $T=0$ solution from eq. (3.2) (since the finite-T corrections to the kernel are subdominant) and inserting in the free action (2.4), the spacetime volume eventually factorizes and we can compute the effective action density, or effective potential, $V_{\text {eff }}(a)$ of the constant background field a. To remove irrelevant field-independent divergences, we also subtract the contribution from $\mathrm{a}=0$; for further details, see Ref. [8]. 

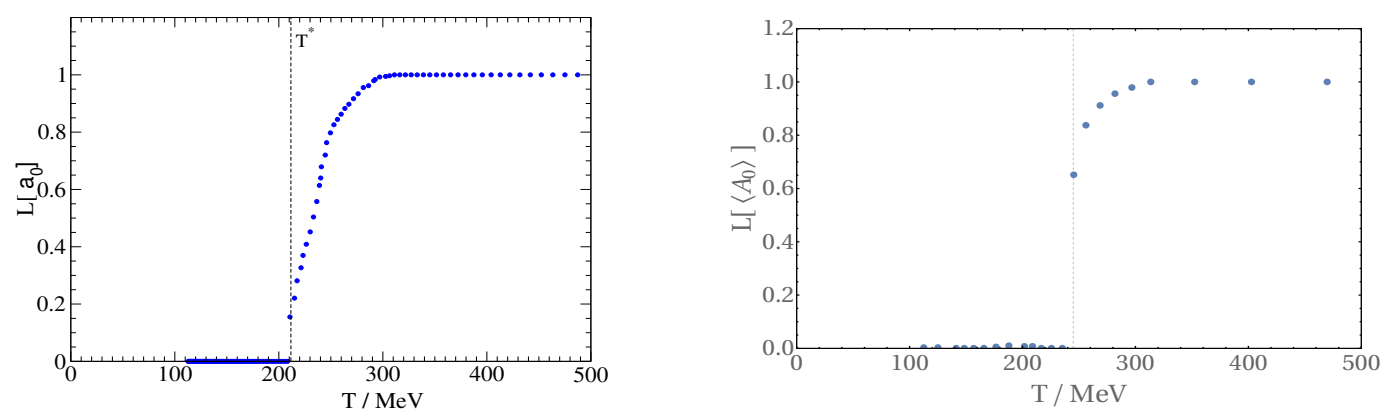

Figure 3: Expectation value of the Polyakov loop as a function of temperature for the colour groups $S U(2)$ (left) and $S U(3)($ right).

From the background field $a_{\min }$ which minimizes the effective potential $V_{\text {eff }}(a)-V_{\text {eff }}(0)$, we can infer the Polyakov loop

$$
\langle\operatorname{tr} L\rangle=\langle\operatorname{tr} \exp (\ln L)\rangle \approx \exp [\langle\operatorname{tr} \ln L\rangle]=\exp [-\beta a] .
$$

This is plotted in Fig. 3 for the colour groups $S U(2)$ and $S U(3)$. As can be clearly seen, there is a phase transition from a confined phase with $\langle\mathrm{L}\rangle=0$ for $T<T^{*}$ to a deconfined phase in which $\langle\mathrm{L}\rangle \neq 0$ quickly approaches unity. In the left panel for $G=S U(2)$, the transition is 2 nd order, while it is clearly discontinuous (1st order) for the case $G=S U(3)$ in the right panel. The critical temperatures are predicted as

$$
\begin{array}{llr}
S U(2): & T^{*}=216 \mathrm{MeV} & \text { lattice }: T^{*}=306 \mathrm{MeV} \\
S U(3) & T^{*}=245 \mathrm{MeV} & \text { lattice }: T^{*}=284 \mathrm{MeV},
\end{array}
$$

where we have also listed the corresponding lattice results from Ref. [13]. The temperatures are in good agreement to the lattice data, in particular if one considers that the overall mass scale has been fixed entirely in the $T=0$ renormalization, and all results here are parameter-free predictions.

\section{Thermodynamics}

In Ref. [9], we have studied the equation of state of the Yang-Mills plasma at finite temperature within the covariant variational approach. The central quantity is the free action eq. (2.4), or rather its minimum under variation of our (Gaussian) ansatz,

$$
F(\beta)=\min _{\mu} F_{\beta}(\mu)=\min _{\mathrm{a}} \Gamma_{\beta}[\mathrm{a}]=-\ln Z(\beta)=V_{3} \beta \cdot f(\beta) .
$$

Since the confined phase has an instability against the formation of a non-trivial background field $a \neq 0$ (see above), we must include in our variation Gaussian measures with and without background field $\left\langle A_{0}\right\rangle=\mathrm{a} \delta_{\mu 0}$. The minimum of the corresponding effective potential thus gives the effective action from which the spacetime volume $V_{4}=V_{3} \beta$ factorizes because of translational invariance. The remainder $f(\beta)$ is hence the free action per unit spacetime volume, or the free energy density. From this quantity, we can derive other interesting thermodynamical observables such as the pressure $p(\beta)=-f(\beta)$ or the energy density $\varepsilon(\beta)=f(\beta)+\beta \partial f / \partial \beta$. 

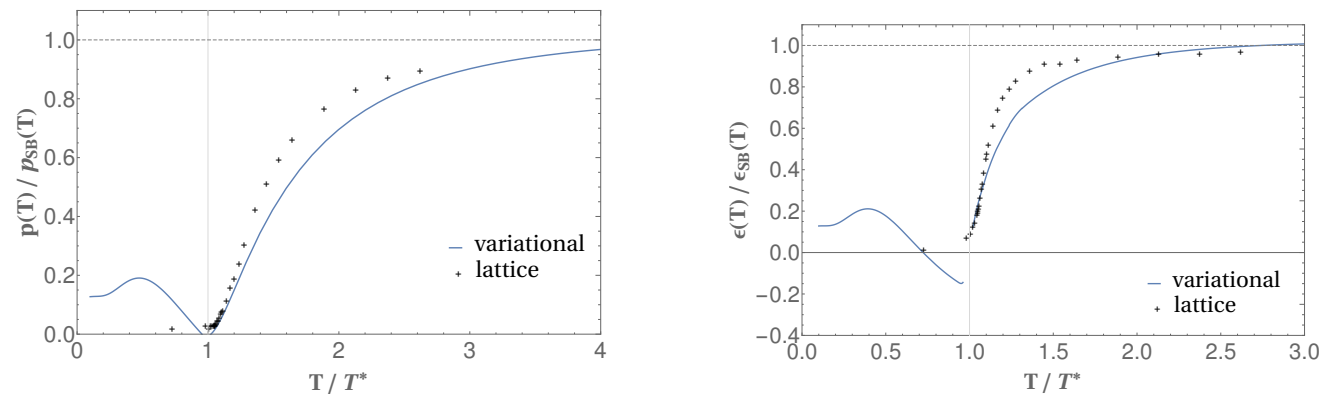

Figure 4: Pressure (left) and energy density (right) for $G=S U(2)$ Yang-Mills theory from the variational approach, compared to lattice date taken from [11].

As explained above, the free energy density is simply obtained from the minimum of the effective potential. In contrast to the calculation of the Polyakov loop, where we subtracted the $\mathrm{a}=0$ contribution, we must now include all degrees of freedom. This means that the free energy density and the pressure will diverge. The origin of this divergence is, however, well understood: It is simply the temperature-independent vacuum energy present in any quantum field theory, which must be removed by a cosmological constant counter term. In Ref. [9], we have shown that this can be done most succinctly by Poisson resummation of the Matsubara series in the free action. The result is an expression for the free energy density in a background (4.2) of the form $(G=S U(2))$

$$
\beta^{4} f_{\beta}(x)=-\frac{2}{\pi^{2}} \sum_{v=1}^{\infty} \frac{\cos (2 \pi v x)+\frac{1}{2}}{v^{4}} h(\beta v) .
$$

The subtraction of the vacuum energy corresponds simply to the omission of the divergent contribution $v=0$ in the Poisson sum. The function $h(\lambda)$ is related to the dispersion relation of all active degrees of freedom, cf. Ref. [9] for details.

In Fig. 4, we present the pressure and energy density computed from eq. (5.2) using our variational solution $\omega(k)$. As can be seen, the correct Stefan-Boltzmann limit is approached at $T \rightarrow \infty$ in both functions. In the entire deconfined region, the variational approach gives a good description of the lattice data from Ref. [11], although the pressure and energy density are slightly underestimated. There is a clear phase transition at which the pressure drops to zero, $p\left(T^{*}\right)=0$. In the confined phase, the only excitations are glueballs whose contributions to the partition function are exponentially suppressed, since the lightest glueball has a mass of arond $1.5 \mathrm{GeV}$. One would therefore expect nearly zero pressure and energy density throughout the entire confinement region, and the lattice data corroborates this reasoning. By contrast, the variational approach has all kind of defects in the confined region: The pressure increases with decreasing temperature to a shallow maximum and then settles for a non-vanishing limit indicating that the ansatz still exhibits massless particles (ghosts) in this region. Even more problematic is the energy density, which jumps at $T^{*}$ and even becomes negative for a substantial portion of the confined phase.

Similar defects have been seen in practically all functional approaches, cf. [14]. The physical reason behind it is that the effective degrees of freedom in these approaches are always (constituent) gluons and ghosts. To describe the proper physics in the confined region, functional approaches would have to be rich enough to describe the formation of colourless glueballs out of these constituents. (It is clear that a Gaussian ansatz is not able to do this.) Instead, confinement comes 
about through ghost dominance, i.e. through an abundance of enhanced ghost degrees of freedom in the deep infrared. While this is sufficient to restore center symmetry for the Polyakov loop, it necessarily leads to the wrong thermodynamics. A more complete description would have to devise a method to form glueballs dynamically within a variational ansatz, or to include them as effective degrees of freedom from the outset.

\section{Non-Gaussian measures}

So far, all our investigations have been performed with the simple Gaussian ansatz eq. (3.1). To conclude this talk, I will briefly present a method to extend the variational approach beyond this limitation by using Dyson-Schwinger equations (DSEs). It should be noted that, for any reasonable theory, the tower of DSEs must be truncated. This means that we are always in a situation as in the right panel of Fig. 1, where the theories covered by truncated DSEs with variational vertices will not include the original theory (or there would be no need for the truncation). The variational approach can then be used to optimize the vertices of the DSE to give the best description for a given truncated set of DSEs.

As a simple example for this technique, let us look at the ghost dominance model for YangMills theory, which is defined by a trial Ansatz action

$$
R=\frac{1}{2} \omega(3,4) A(3) A(4)+\bar{c}(c)\left[-\Delta(3,4)+\Gamma_{0}(3,4, ; 5) A(5)\right] c(4)
$$

Here, we have used a convenient shortcut notation where the same digits in an expression indicate a sum over all discrete indices and an integration over all spacetime arguments. The ghost-gluon coupling in the last term is cubic in the fields so that $R$ is non-Gaussian. (We have taken the vertex to be bare in accordance with the rainbow-ladder approximation discussed earlier.) The DysonSchwinger equations derived from the action $R$ now also involve a seprate equation for the triple gluon vertex, which was missing entirely in the Gaussian ansatz:

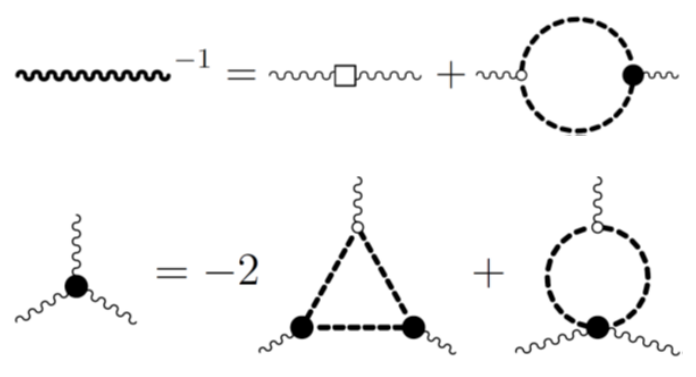

Here, fat vertices and lines are fully dressed, and the open square represent the variational kernel $\omega$ (which is no longer the inverse gluon propagator). From the gap equation written in the form

$$
\left\langle\frac{\delta R}{\delta \omega}(S-R)\right\rangle_{R}=\left\langle\frac{\delta R}{\delta \omega}\right\rangle_{R}\langle S-R\rangle_{R}
$$


we can derive a diagramatical representation which looks quite formidable

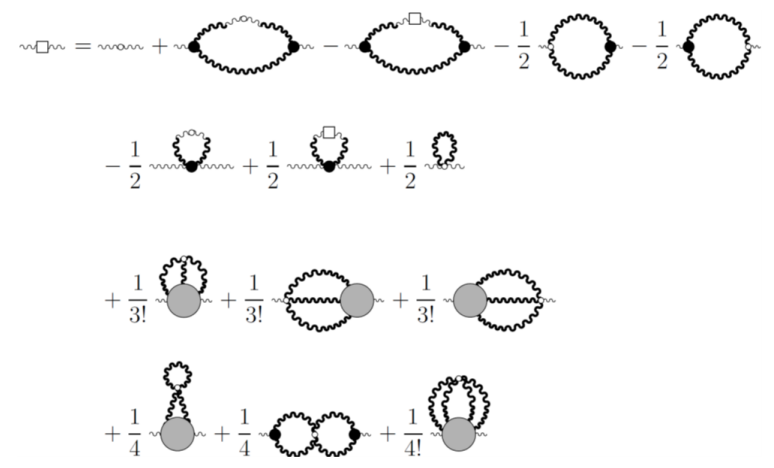

The last two lines are two-loop contributions that are neglected in the usual truncation schemes.

\section{Summary and Conclusion}

In this talk I have presented the foundations of the covariant variational approach to YangMills theory and its extension to finite temperature. While most thermal properties are reproduced accurately (including the deconfinement phase transition), the thermodynamics in the confined phase point to shortcomings of the Gaussian approach which cannot describe the correct glueball degrees of freedom. Finally, I have briefly discussed how the covariant variational approach can be combined with Dyson-Schwinger techniques to go beyond the Gaussian ansatz, and illustrated it with a simple ansatz for Yang-Mills theory. In the future, we plan to apply the same techniques to full QCD including fermions, which would allow to study the QCD phase diagram in the regions where lattice calculations currently fail.

\section{References}

[1] J.M. Pawlowski, Annals Phys. 322, 2831 (2007); H. Gies, Lect. Notes Phys. 852, 287 (2012).

[2] R. Alkofer and L. von Smekal, Phys. Rept. 353, 281 (2001). C. S. Fischer, J. Phys. G32, R253 (2006);

[3] M. Tissier and N. Wschebor, Phys. Rev. D82, 101701 (2010).

[4] D. Zwanziger, Nucl. Phys. B399, 477 (1993).

[5] C. Feuchter and H. Reinhardt, Phys. Rev. 70, 105021 (2004);

[6] M. Quandt, H. Reinhardt and J. Heffner, Phys. Rev. 89, 065037 (2014).

[7] M. Quandt and H. Reinhardt, Phys. Rev. D92, 025051 (2015).

[8] M. Quandt and H. Reinhardt, Phys. Rev. D94, 065015 (2016).

[9] M. Quandt and H. Reinhardt, Phys. Rev. D96 (2017) 054029.

[10] I. Bogolubsky et al., Phys. Lett. B676, 69 (2009).

[11] J. Engels et al. Z. Phys. C42 (1989) 341; F. Karsch and E. Laermann, arXiv:hep-lat/0305025 (2003).

[12] J. Braun, H. Gies and J. M. Pawlowski, Phys. Lett. B684, 262 (2010).

[13] B.Lucini, M.Teper and U.Wenger, J. High Energy Phys. 01, 061 (2004).

[14] F.E. Canfora et al., Eur. Phys. J. C75 (2015) 326; C.S. Fischer and J. Luecker, Phys. Lett. B718

(2013) 1036; K.-I. Kondo, arXiv:1508.02656 (2015); Reinosa et al., Phys. Rev. D91 (2015) 045035. 